Research Paper

\title{
Localization of Human Mesenchymal Stem Cells from Umbilical Cord Blood and Their Role in Repair of Diabetic Foot Ulcers in Rats
}

\author{
Qing-Song Zhao ${ }^{1 *}$, Nan Xia ${ }^{1 *}$, Nan Zhao ${ }^{1}$, Ming $\mathrm{Li}^{1}$, Chang-Long Bi ${ }^{1}$, Qing Zhu ${ }^{1}$, Guo-Fen Qiao ${ }^{\circledR}$, \\ Zhi-Feng Cheng ${ }^{1 凶}$ \\ 1. Department of endocrinology and Metabolism, the Fourth affiliated Hospital of Harbin Medical University \\ 2. Department of Pharmacology, Harbin Medical University; Harbin, China \\ * These authors contributed equally to this work
}

\begin{abstract}
$\square$ Corresponding authors: Z-F Cheng (czfusa2013@163.com), Department of endocrinology and Metabolism, the Fourth affiliated Hospital of Harbin Medical University, \#37 Yi-Yuan Street, Nan-Gang District, Harbin 150001 China; Tel.: 189-0360-2198; fax: +86 451-8257-6770, or G-F Qiao (qiaogf88@163.com), Department of Pharmacology, Harbin Medical University, \#157 Bao-Jian Road, Harbin 150081 China; Tel.: +86 451-8667-1345, Fax: +86 451- 8666-7511
\end{abstract}

(c) Ivyspring International Publisher. This is an open-access article distributed under the terms of the Creative Commons License (http://creativecommons.org/ licenses/by-nc-nd/3.0/). Reproduction is permitted for personal, noncommercial use, provided that the article is in whole, unmodified, and properly cited.

Received: 2013.07.23; Accepted: 2013.12.04; Published: 2013.12.19

\begin{abstract}
The aim of this study is to explore the localization of human mesenchymal stem cells from umbilical cord matrix (hMSCs-UC) and the role of these cells in the repair of foot ulcerate tissue in diabetic foot ulcers in rats. A diabetic rat model was established by administering Streptozotocin. Diabetic foot ulceration was defined as non-healing or delayed-healing of empyrosis on the dorsal hind foot after 14 weeks. hMSCs-UC were delivered through the left femoral artery. We evaluated the localization of hMSCs-UC and their role in tissue repair in diabetic foot ulcers by histological analysis, PCR, and immunohistochemical staining. A model for diabetes was established in 54 out of 60 rats ( $90 \%$ success rate) and 27 of these rats were treated with hMSCs-UC. The area of ulceration was significantly and progressively reduced at 7 and 14 days following treatment with hMSCs-UC. This gross observation was strongly supported by the histological changes, including newly developed blood vessels and proliferation of inflammatory cells at 3 days post-treatment, significant increase in granulation tissue at 7 days post-treatment and squamous epithelium or stratified squamous epithelium at 14 days post-treatment. Importantly, human leukocyte antigen type-I (HLA-I) was confirmed in ulcerated tissue by RT-PCR. The expression of cytokeratin 19 was significantly increased in diabetic model rats, with no detectable change in cytokeratin 10. Additionally, both collagens I and III increased in model rats treated with hMSCs-UC, but the ratio of collagen I/III was less significant in treated rats compared with control rats. These results suggest that hMSCs-UC specifically localize to the target ulcerated tissue and may promote the epithelialization of ulcerated tissue by stimulating the release of cytokeratin 19 from keratinocytes and extracellular matrix formation.
\end{abstract}

Key words: human mesenchymal stem cell from umbilical cord matrix, human leukocyte antigen, diabetic foot, tissue repair

\section{Introduction}

Diabetes is a widespread medical problem in modern society. In China, 92.4 million patients have been diagnosed with diabetes, and 148.2 million adults with prediabetes have been reported (1). Diabetic foot ulceration, which dramatically affects quality of life and can be life-threatening, is an important 
complication of diabetes (2). The incidence of diabetic foot ulceration patients with diabetes is approximately $1.7-11.9 \%$, according to various investigations (3). About $15 \%$ of patients with diabetes have a tendency to develop diabetic foot ulcers throughout life (4), and therefore it is an important consideration in the clinical management of diabetes.

Published data have demonstrated that mesenchymal stem cell (MSCs) can be introduced into cardiomyocytes, which opens up a new research direction for the treatment of myocardial infarction (5). MSCs have also been shown to repair glomerular cells damaged by acute renal failure, which could be of clinical significance in the restoration of renal function (6). In another study, investigators transformed MSCs into neurons under certain conditions $(7,8)$. All these findings provide potential clinical use for MSCs, which might be applied to the treatment of diabetic foot ulceration; however, very little research has focused on the underlying mechanisms of cellular repair in diabetic foot ulcers.

In this study, we injected hMSCs-UC via the left femoral artery in a rat model for diabetes. We assessed the mobilization and localization of hMSCs-UC in diabetic foot ulcers using antigenic differences in HLA-1 between human and rat. To further understand the potential mechanisms of repairing diabetic foot ulcers, we analyzed matrix (collagen I \& III) and cytokeratins (CKs) 10 and 19 released by MSCs. We demonstrated that hMSCs-UC localized superficially in ulcerated tissue, promoting the release of CKs and stimulating tissue repair in diabetic foot ulcers.

\section{Methods}

\section{Experimental animals}

Adult male Sprague-Dawley (SD) rats (180-220 g) were purchased from Chinese Shanghai SLAC Laboratory Animal Co Ltd (grade: clean, license: SCXK 2011-0005) and used to establish a rat model for diabetes. All rats were maintained on a 12-hour light cycle in the animal facility of the second affiliated Hospital of Harbin Medical University. Experimental protocols used in this experiment were previously approved by the Institutional Animal Care and Use Committee of the School of Medical Science, Harbin Medical University. All animal experiments were conducted in accordance with the "Principles of Laboratory Animal Care" (NIH publication no. 85-23, revised 1985; http://grants1.nih.gov/grants/olaw/ references/phspol.htm), as well as with specific national laws where applicable.

\section{Reagents, software, equipment}

Streptozotocin (STZ, Sigma, St Louis, MO, USA) was use to induce diabetes in the rat model.
Blood-glucose meter and test strips were purchased from Bayer Medical Care Co Ltd (Leverkusen, Germany). Blood glucose levels in diabetic rats were maintained by injection of protamine zinc insulin (Wan-Bang Biochemical Medicine Co. Ltd., Xuzhou, China). The hMSCs-UC was provided by Jing-Meng Stem Cell Technology Co. Ltd. (Beijing, China). All other chemicals for cell culture were purchased directly from routine commercial sources.

Primers for HLA-I (Sheng-Gong Biotech, Shanghai, China) were used in RT-PCR. For immunohistochemistry, we used polyclonal rabbit anti-rat collagen type-1, collagen type-III, and CK10 antibodies (Boster Biological Technology, Wuhan, China) and monoclonal rat anti-human CK19 antibody (ZSGB Biotech, Beijing, China). Moloney murine leukemia virus reverse transcriptase (Mo-MLVRT; Sheng-Gong Biotech, Shanghai, China) was prepared at a dilution of 50 units $/ \mu$ in $5 x$ Mo-MLVRT buffers, with oligo $d$ (T) and OD: 1:0. Histological/pathological analysis was carried out using imaging software (BA2000; Motic, Richmond, Canada). Other equipment utilized included a MyCycler ${ }^{\mathrm{TM}}$ PCR (Bio-Rad, Hercules, USA), a gel imaging analysis system (GIS-3000, Hema Medical Instruments, Guangdong, China), and a cell culture incubator (Binder, Tuttlingen, Germany).

\section{Model for diabetic foot ulceration in rats}

Sixty rats were fasted and deprived of water for 10 hours, injected once intraperitoneally with 55 $\mathrm{mg} / \mathrm{kg}$ of STZ, and then given free access to food and water. One week after injection with STZ, we used the following criteria to select successful rat models for diabetes (1) fasting blood glucose over $16.7 \mathrm{mM}$; (2) glucose levels well-controlled $(20-25 \mathrm{mmol} / \mathrm{l})$ by $2-12$ units/day of insulin. To create a model for diabetic foot ulcers, we made a $4 \times 4 \mathrm{~mm}$ empyrosis on the dorsal hind foot of the diabetic rats at 10-12 weeks after treatment with STZ. To create a successful model for diabetic foot ulcers, this procedure was repeated until the ulceration/wound induced by empyrosis did not heal, or demonstrated delayed healing, for at least 14 weeks $(9,10)$.

\section{Preparation of hMSCs-UC}

hMSCs-UC were cultured in medium containing 90\% DMEM-F12 (Sigma, St Louis, USA), 10\% umbilical cord blood plasma collected from different individuals with an identical blood type (Sigma, USA), 2.0 $\mathrm{mmol} / 1$ glutamine (Sigma, USA), 100 units/ml penicillin-streptomycin-neomycin (PSN) antibiotic mixture (Sigma, USA), $5 \mathrm{ng} / \mathrm{ml}$ epidermal growth factor (EGF, Sigma, USA), and $2 \mathrm{ng} / \mathrm{ml}$ of fibroblast growth factor (bFGF, Sigma, USA). hMSCs-UC were stored at $-80^{\circ} \mathrm{C}$ and were recovered by transferring to a $37^{\circ} \mathrm{C}$ 
water bath with gentle shaking, followed by centrifugation at $37^{\circ} \mathrm{C}$. The supernatant was discarded and the hMSCs-UC were re-suspended in $5 \mathrm{ml}$ complete culture medium, transferred into tissue culture flasks, and cultured at $37^{\circ} \mathrm{C}, 5 \% \mathrm{CO}_{2}$ in a humidified environment for 24 hours.

For continuous cell culture of hMSCs-UC, the adherent hMSCs-UC were washed with $\mathrm{Ca}^{2+}$-free and $\mathrm{Mg}^{2+}$-free PBS and transferred into a petri dish containing culture medium with $0.05 \%$ trypsin-EDTA at $\sim 22^{\circ} \mathrm{C}$ for $1-2 \mathrm{~min}$. An equal volume of culture medium was added and gentle aspiration was used to separate cells. This cell suspension was transferred into a $15 \mathrm{ml}$ tube and centrifuged at $1200 \mathrm{rpm}$ for 5 min. The supernatant was removed and the pallet of hMSCs-UC was then re-suspended in the appropriate volume of culture medium.

$6.0 \times 10^{3} / \mathrm{cm}^{2}$ of hMSCs-UC were plated onto petri dishes for continuous cell culture at $37^{\circ} \mathrm{C}, 5 \%$ $\mathrm{CO}_{2}$ in a humidified environment. Under these culture conditions, cells grew to confluence by 3-4 days, and further passage was required before using the cells. For identification of particular biological properties of MSCs, flow cytometry was used to detect and quantify expression of CD44, CD73, and CD90 (Figure $1)$. The details for cytometry testing are: (1) hUC-MSCs were digested by trypsin $(0.05 \%+0.04 \%$ EDTA); (2) Cell suspension was centrifuged at 1400 rmp for $10 \mathrm{~min}$; supernatant was discarded and re-suspended by using $300 \mu \mathrm{l}$ PBS; (3) Every $100 \mu \mathrm{l}$ of above cell suspension was labeled as negative control, sample 1 , and sample 2 and incubated with antibodies (see details below) at room temperature $\left(\sim 22^{\circ} \mathrm{C}\right)$ avoiding light exposure for $20 \mathrm{~min}$. Before cytometry testing, $2 \mu \mathrm{l}$ IgG1-FITC and IgG1-PE was added into negative control; $2 \mu$ l CD90-FITC, CD105-Percp, CD-44-PE, and HLA-DR-APC were added into sample 1; $2 \mu \mathrm{l} \mathrm{CD29-APC,} \mathrm{CD45-FITC,} \mathrm{and} \mathrm{CD73-PE} \mathrm{were}$ added into sample 2 . All antibodies used in this testing are rat against human antibody (BD Biosciences, Franklin Lake, NJ, USA); (4) $1.0 \mathrm{ml}$ PBS was added into each tube and centrifuged at $1200 \mathrm{rmp}$ for $6 \mathrm{~min}$; and then discarded the supernatant and added another $400 \mu \mathrm{l}$ PBS for re-suspension for cytometry testing (FACScalibur ${ }^{\mathrm{TM}}$; BD Biosciences; Franklin Lake, NJ, USA).
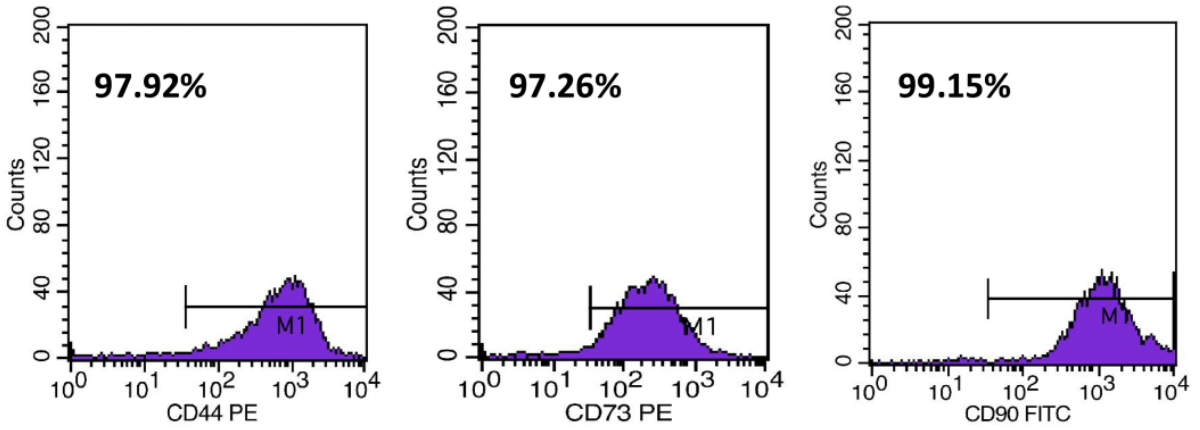

Figure I: Identification of CD44, CD73, and CD90 expression in hMSCs-UC measured by flow cytometry. The percentages were automatically calculated during the measurement.

\section{Administration of hMSCs-UC to diabetic rats}

Twenty-seven diabetic model rats with foot ulceration were randomly selected and anesthetized with and intraperitoneal injection of $4 \%$ chloral hydrate (Tianjin Chemical Reagent Co., Tianjin, China) at $0.5 \mathrm{ml} / 100 \mathrm{~g}$ body. After the rats were completely relaxed, we bluntly dissected the left femoral artery and administered $2.0 \times 10^{6} \mathrm{hMSC}-\mathrm{UC} /$ rat through left femoral artery. The rats with diabetic foot ulcers were sacrificed at 3 days (D3), 7 days (D7), and 14 days (D14) after administration of hMSCs-UC. After sacrificing the rats, the feet with diabetic ulcers were collected and cut vertically along the center line of ulceration. Half of each foot was stored immediately at $-80^{\circ} \mathrm{C}$ for detection of human leukocyte antigen-I (HLA-I) by RT-PCR, and the other halves were fixed in $4 \%$ chloral hydrate for hematoxylin and eosin (HE) staining and immunohistochemical analysis.

\section{Detection of HLA-I by RT-PCR}

For total mRNA extraction, ulcerated tissues (weight $100 \mathrm{mg}$ ) were ground with liquid nitrogen, then add $800 \mathrm{ml}$ TRIzol ${ }^{\circledR}$, and $300 \mathrm{ml}$ of $\mathrm{CHCl}_{3}$, shake well and centrifuge at 12,000 rpm for $10 \mathrm{~min}$. Supernatant $(600 \mathrm{ml})$ was collected, add equal volume of isopropyl alcohol, and the mixture was centrifuged again under the same conditions; Removed the supernatant, the precipitate was washed by $600 \mathrm{ml}$ of $75 \%$ ethanol prepared with Diethylpyrocarbonate (DEPC) water, and centrifuge for an additional 10 min. Discarded the supernatant and the ethanol was evaporated at RT for $5 \mathrm{~min}$, then the precipitate was dissolved by $21 \mu \mathrm{LEPC}$ water. For reverse transcrip- 
tion: I $\mu$ g total mRNA with $1.0 \mu$ l of oligo d (T) was transferred into a clean tube at $70^{\circ} \mathrm{C}$ for $5 \mathrm{~min}$, the extracted mRNA was reverse transcribed to a cDNA template with $1.0 \mu \mathrm{l} \mathrm{dNTP}, 0.5 \mu \mathrm{l}$ RNase Inhibitor, 0.5 $\mu \mathrm{l}$ reverse transcriptase, and $5 \mu \mathrm{l}$ buffer. For cDNA amplification by RT-PCR, glyceraldehyde 3-phsophate dehydrogenase (GAPDH, forward: 5'-ACATACTCAGCACCAGCATCACC-3' and reverse: 5'-CAAGTTCAACGGCACAGTCAAGG-3') was used as an internal control. The sequences for both forward and reverse primers of HLA-I (NM_002116.7) were 5'-GCCTACGACGGCAAGGAT TAC-3' and 5'-ATGGTGGGCTGGGAAGACAG-3', respectively (Sheng-Gong Biotechnology, Shanghai, China). The protocol for PCR amplification was set at $94^{\circ} \mathrm{C}$ for $1 \mathrm{~min}(1 \mathrm{cycle})$, followed by $94^{\circ} \mathrm{C}$ for $30 \mathrm{sec}$, $-59^{\circ} \mathrm{C}$ for $30 \mathrm{sec}$, and $-73^{\circ} \mathrm{C}$ for $30 \mathrm{sec}$ for 25 cycles, and then $72^{\circ} \mathrm{C}$ for $7 \mathrm{~min}$ (I cycles). The product size of HLA-I was $497 \mathrm{bp}$.

\section{Histological analysis of diabetic foot ulcerative tissue}

Tissue was collected from either control or treated rats, fixed with $4 \%$ paraformaldehyde for 48 hours, embedded in paraffin, and sectioned at $4.0 \mu \mathrm{m}$. After de-waxing twice for 5 minutes with dimethylbenzene, the tissue slices were dehydrated by successive concentrations of ethanol $(100 \%, 95 \%, 90 \%$, and $80 \%$ ) for 5 minutes at each concentration. Finally, the tissues were washed twice in distilled water for 5 minutes each wash. After hematoxylin staining for 5 minutes, tissue slices were washed in water, treated with $0.1-1.0 \%$ hydrogen ethanol for 30 minutes and immersed in water for 15 minutes. The tissues were then H\&E stained for 5 minutes, washed for 3 minutes, dehydrated and tissue transparent again with ethanol $(80 \%, 90 \%, 95 \%$ and $100 \%)$ for 5 minutes each and dimethylbenzene for 15 minutes. Finally, the tissue slices were sealed rapidly with nature balsam and cover slips for microscopic analysis.

\section{Immunohistochemical analysis of diabetic foot ulcerative tissue}

After fixing, embedding in paraffin, and dewaxing, the tissue slices were blocked using 3\% $\mathrm{H}_{2} \mathrm{O}_{2}$. For Immunostaining, the tissue was incubated with primary antibodies (primary) against collagen type-1 (1:100 dilution), collagen type-III (1:50 dilution), CK10 (1:50 dilution), and CK19 (1:100 dilution), at $4{ }^{\circ} \mathrm{C}$ overnight, respectively, followed by anti-rabbit IgG (secondary) for 20 minutes. After hematoxylin staining, tissue slices were washed for 10 minutes and then dehydrated with ethanol, treated with dimethylbenzene, and sealed for microscopic analysis (11-13).

\section{Data acquisition and statistical analysis}

In this study, the levels of expression of collagens and CKs at different time points in control and treated groups were quantified by standard validation as described in detail previously (14). The expression levels were presented as negative expression $(-)$, positive expression $<25 \%(+/-)$, positive expression $<50 \%(+)$, positive expression $<75 \%(++)$, and positive expression $>75 \%(+++)$. The size of ulcerative area was measured as the product of the shortest and longest axes of the ulcer and presented in $\mathrm{mm}^{2}$. The average data were expressed as mean \pm SD. Data were analyzed by the Wilcoxon rank-sum test, the Kruskal-Wallis $\mathrm{H}$ test, the chi-square test, and the $t$-test where appropriate using SAS v9.1 software. $P$ values less than 0.05 between the control and treated groups were considered to be statistically significant.

\section{Results}

\section{Rat Model for Diabetic Foot Ulceration}

The model for diabetic food ulceration was duplicated in 54 out of 60 rats ( $90 \%$ success rate). Half of the model rats $(n=27$, treated group) received hMSCs-UC $\left(2.0 \times 10^{6}\right.$ cells/rat $)$. The other half of the model rats ( $n=27$, control group) were treated with the same volume of saline. At day 3 (D3) post-treatment, the size of the foot ulcers were somewhat enlarged in the group that had received hMSCs-UC (Figures $2 \& 3,24.8 \pm 4.2 \mathrm{~mm} 2$ in the control group vs. $28.6 \pm 3.4 \mathrm{~mm}^{2}$ in the treated group). However, by day 7 (D7) post-treatment, the size of the foot ulcers was significantly reduced in the group that received hMSCs-UC $\left(26.6 \pm 4.1 \mathrm{~mm}^{2}\right.$ in the control group vs. $21.2 \pm 3.25 \mathrm{~mm}^{2}$ in the treated group, $P<$ 0.01). By day 14 (D14) post-treatment, the size of foot ulcers in the mice treated with hMSCs-UC were further reduced $\left(27.7 \pm 4.8 \mathrm{~mm}^{2}\right.$ in the control group vs. $14.7 \pm 3.9 \mathrm{~mm}^{2}$ in the treated group, $\left.P<0.001\right)$. In the control group, the size of the foot ulcers increased further during the 14 days of observation.

\section{Identification and Localization of hMSCs-UC}

We analyzed the expression of HLA-I, as an indicator of the presence of hMSCs-UC, in ulcerative tissue compared with the expression in liver and kidney in the same rats (Supplemental Figure 1). Expression of HLA-I was also compared among the model control rats $(\mathrm{C})$ that had received saline and the hMSCs-UC treated model rats. The localization of HLA-1, as an indicator of hMSCs-UC, was monitored for 14 days after hMSCs-UC were administered to the treated group (Figure 4). Our results demonstrated the expression of HLA-I in diabetic foot ulcers at 3 days (D3), 7 days (D7), and 14 days (D14) after treat- 
ment with hMSCs-UC. The expression levels of HLA-I were 10-50 fold higher compared with the control group, and HLA-I expression was not detected in the model group. Under the same experimental conditions, HLA-I expression was not observed in the liver or kidneys at any time points in the treated group (Supplemental Figure 1).
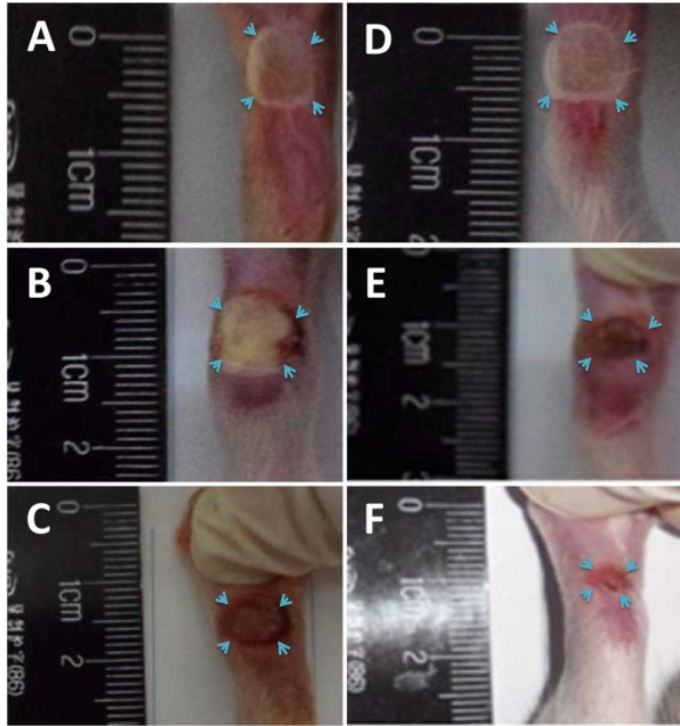

Figure 2: Representative changes in diabetic foot ulceration between human mesenchymal stem cell in umbilical cord blood (hMSCs-UC)-treated and model control groups. (A)-(C): the images were collected from 3 (D3), 7 (D7), and I 4 days (DI4) model control rats, respectively, before treatment with hMSCs-UC; (D)-(F): the images were taken from the same time points of diabetic model rats after hMSCs-UC treatment. The ulcerate area on foot of diabetic rats was indicated by light blue arrowhead and presented as square millimeter $\left(\mathrm{mm}^{2}\right.$, i.e. long axis $\times$ short axis of ulcerate area). The average data were expressed as mean $\pm S D, n=9$ for each group. $* P<0.05$ and $* * P<0.01$ vs model control group.

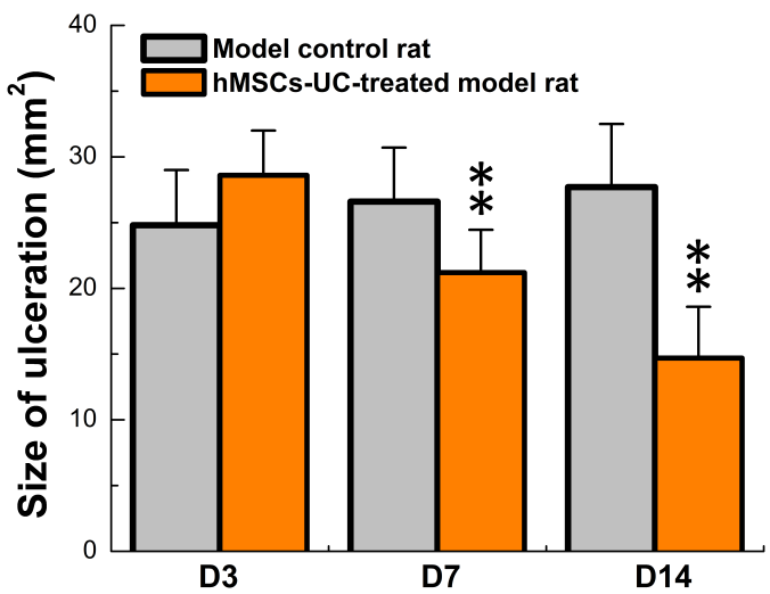

Figure 3: Progressively reduction in the size of diabetic foot ulceration in hMSCs-UC treated model rats during observation compared with model control rats. Data were collected at 3, 7, and I4 days after hMSCs-UC treatment and averaged data were presented as mean $\pm S D, n=27$ for both model control and treated model groups. $* * p<0.01$ vs model control.

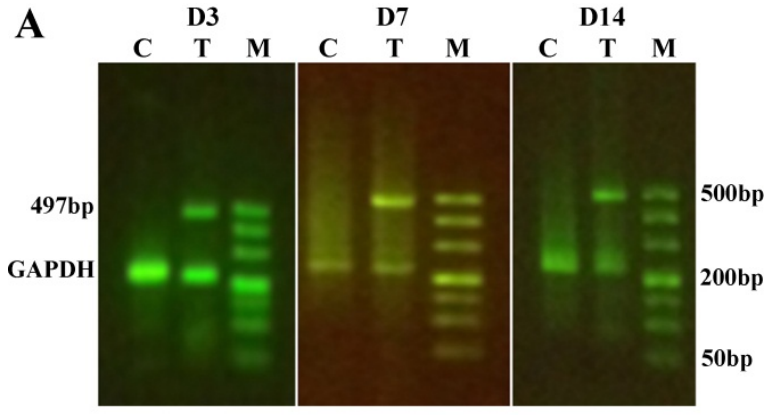

B

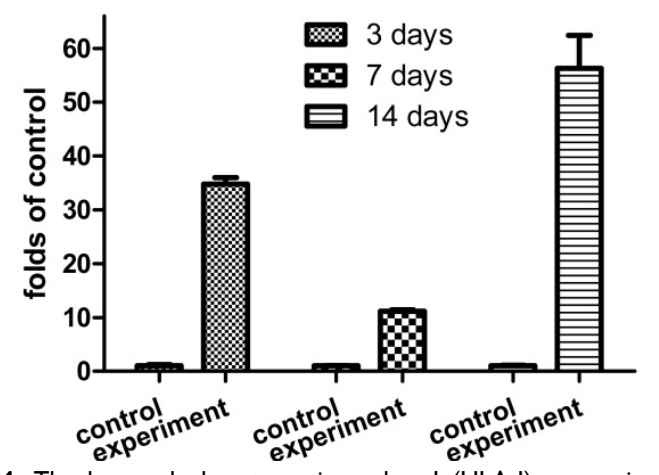

Figure 4: The human leukocyte antigen class I (HLA-I) expression in the ulcerate tissue of diabetic model rats. In treated model group, the model rats were treated with human mesenchymal stem cells in umbilical cord blood (hMSCs-UC) for 3 (D3), 7 (D7), and I4 days (DI4), respectively, and the HLA-I expression level was examined by RT-PCR analysis and compared with model control rats. $(C),(T)$, and $(M)$ present model control with saline, model treated with hMSCs-UC, and maker, respectively. (A): the representative HLA-I protein expression on D3, D7, and DI4 of each group. (B): Summarized data of HLA-I expression as the fold changes. Average data were expressed by fold changes as mean $\pm S D$ and $n=9$ for each group. $* P<0.05$ and $* * P<0.01$ vs model control rats.

\section{Morphological Changes in Diabetic Foot Ulceration}

The expression of HLA-I in ulcerated tissue, but not in other locations such as liver or kidney (Supplemental Figure 1), strongly indicated that the hMSCs-UC are localized specifically to the foot ulcerative tissue. The localization of hMSCs-UC could result in beneficial morphological changes in the repair of diabetic foot ulcers. In H\&E stained tissues, at different time points we observed changes including proliferation of inflammatory cells, newly or aged granulation tissue containing newly developed blood vessels, and simple or stratified epithelium or stratified squamous epithelium (Figure 5). Compared with model control rats (Figure 5A-C), rats treated with hMSCs-UC showed significant increases in inflammatory cells and newly developed or aged granulation tissue with newly developed blood vessels at D3 and D7 post-treatment (Figure 5D \& E, indicated by arrows). Stratified squamous epithelium was also increased at D14 in treated rats (Figure 5F, indicated by *), but was not observed in model rats. 


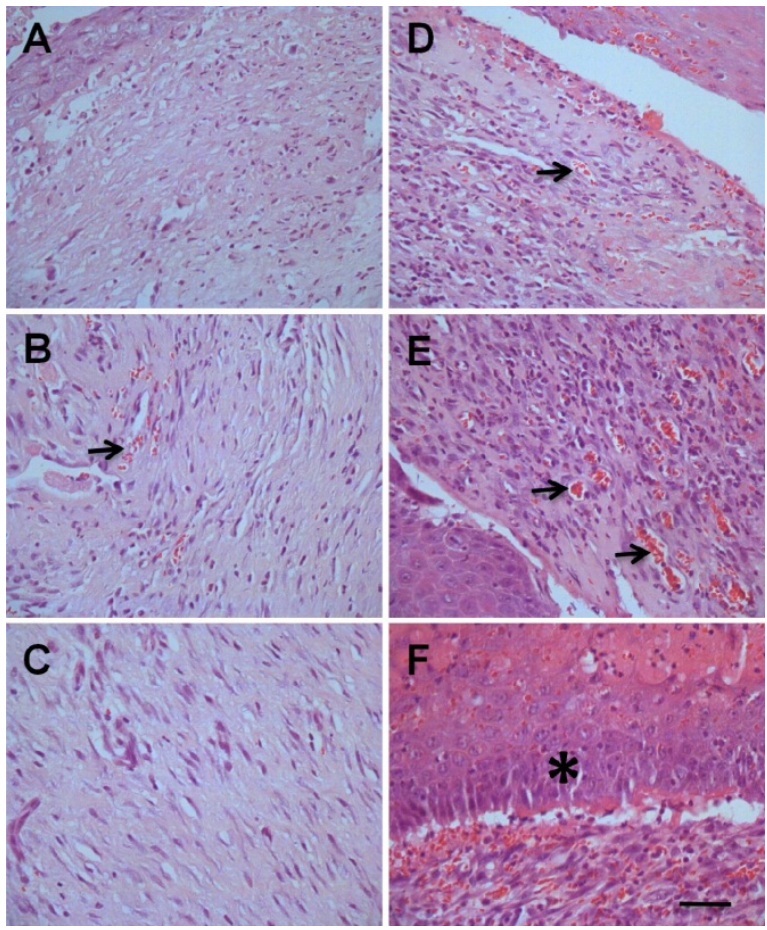

Figure 5: Morphological changes of ulcerate tissue from diabetic foot between hMSCs-UC-treated model and model control groups. In treated group, the diabetic model rats were treated with hMSCs-UC for 3 (D3), 7 (D7), and 14 days (DI4), respectively and the morphological changes, such as inflammatory cells proliferation, newly developed or aged granulation tissue containing newly developed blood vessels (arrows) and fibroblast cells, and simple or stratified squamous epithelium $\left({ }^{*}\right)$, were examined under light microscope $(40 \times) . n=9$ for all groups. (A), (B), and (C): D3, D7, and DI 4 in model control rats, (D), (E), and (F): D3, D7, and DI4 in hMSCs-UC-treated model rats. The scale bar in (F) presents $40 \mu \mathrm{m}$ and also applied for all other images.

\section{Expression of Collagen type-I and type-III}

Collagens are mainly expressed and distributed in the dermis of the skin, and are closely associated with the repair of injured skin. The ratio of collagen type-I/ type-III is another factor to evaluate the healing of ulcerative tissue. Our data showed that the expression of both collagen type-I (Figure 6) and type-III (Figure 7) were markedly increased $(P<0.05$, Table 1$)$ after treatment with hMSCs-UC in a time-dependent manner (Figure 6D-F and Figure 7D-F, indicated by *) compared with model control rats (Figure 6A-C and Figure 7A-C). Importantly, the data also showed that the ratio of collagen type-I/type-III progressively increased in a time-dependent manner in both model control and treated rats at D7 or D14, but the increase was less dramatic in the rats treated with hMSCs-UC. At D7, the collagen type-I/type-III ratio was $1.41 \pm$ 0.18 in the model group and $1.07 \pm 0.15$ in the treated group, and both were increased $(P<0.05$ for both) compared with D3 $(0.96 \pm 0.20$ in the model group and $0.78 \pm 0.15$ in the treated group). The increase in the ratio of collagen type-I/type-III for the treated group was less dramatic compared with the model group $(P$ $<0.05)$. Interestingly, further increase in the collagen
type-I/type-III ratio was statistically significant only in model rats at D14 $(1.94 \pm 0.9, P<0.05$ vs. D7 model). The ratio was slightly increased at D14 in the treated group $(1.12 \pm 0.14)$ but it was not markedly different than the D7 treated group $(P>0.05)$; thus, the collagen type-I/type-III ratio was significantly smaller in the D14 treated group than in the D14 model group $(P<$ 0.01).

\section{Expression of CKIO and CKI 9}

CK10 is the end product of epidermal cell differentiation in the superficial layer of the skin and shows a positive expression in mature skin; therefore, CK10 is a valuable biomarker of functional maturity for epidermal cells. In the current study, expression of CK10 was almost undetectable in ulcerative tissue either in model rats or in hMSCs-UC-treated rats throughout the 14 days after treatment (Table 1). CK19 (Figure 8 ) is secreted by epidermal stem cells located in the basal layer of skin and is temporally expressed in the early stages of epidermal stem cell differentiation; therefore, CK19 could be considered a useful biomarker for epidermal stem cells. Interestingly, CK19 was dramatically expressed in hMSCs-UC-treated rats compared with model rats $(P<0.01)$. In the rats treated with hMSCs-UC, CK19 expression was up-regulated in a time-dependent fashion (Table 1).

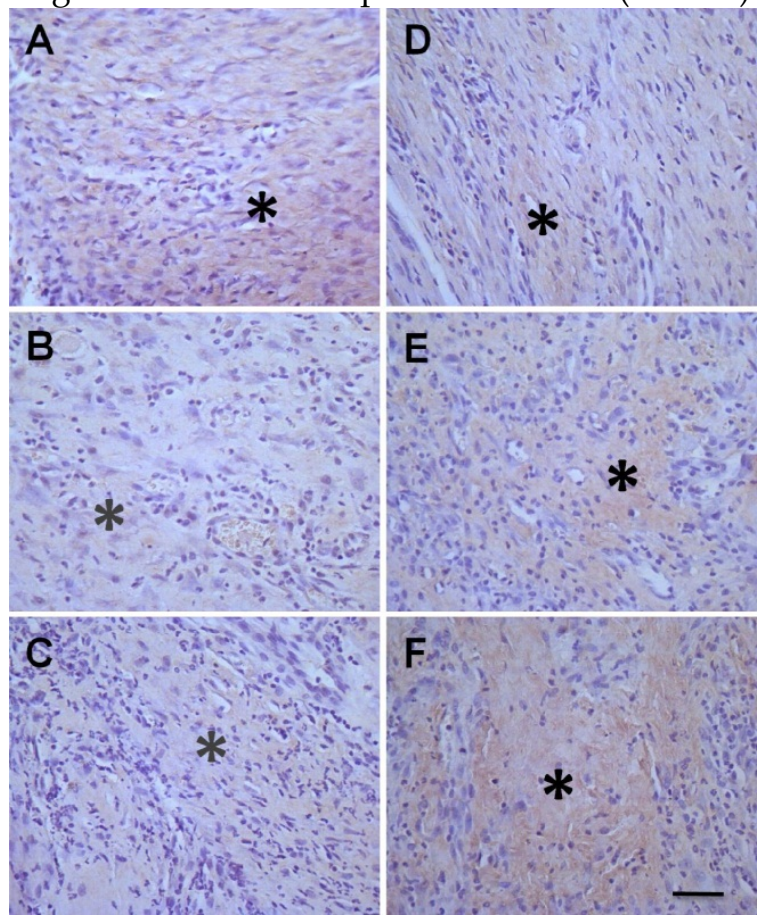

Figure 6: Histological examination of the collagen type-l expression in diabetic foot between treated model and model control groups. In experimental group, the diabetic model rats were treated with human mesenchymal stem cells in umbilical cord blood (hMSCs-UC) for 3 (D3), 7 (D7), and 14 days (DI4), respectively and the collagen type-I expression was examined histologically in all groups. The collagen type-I expression was presented as light-brown (indicated by $\left.{ }^{*}\right)$ under the HE staining. The left column was model control groups with D3 (A), D7 (B), and DI4 (C) and the right column was hMSCs-UC-treated model group at D3 (D), D7 (E), and DI4 (F) after treatment. The scale bar in (F) presents $40 \mu \mathrm{m}$ and also applied for all other images. 

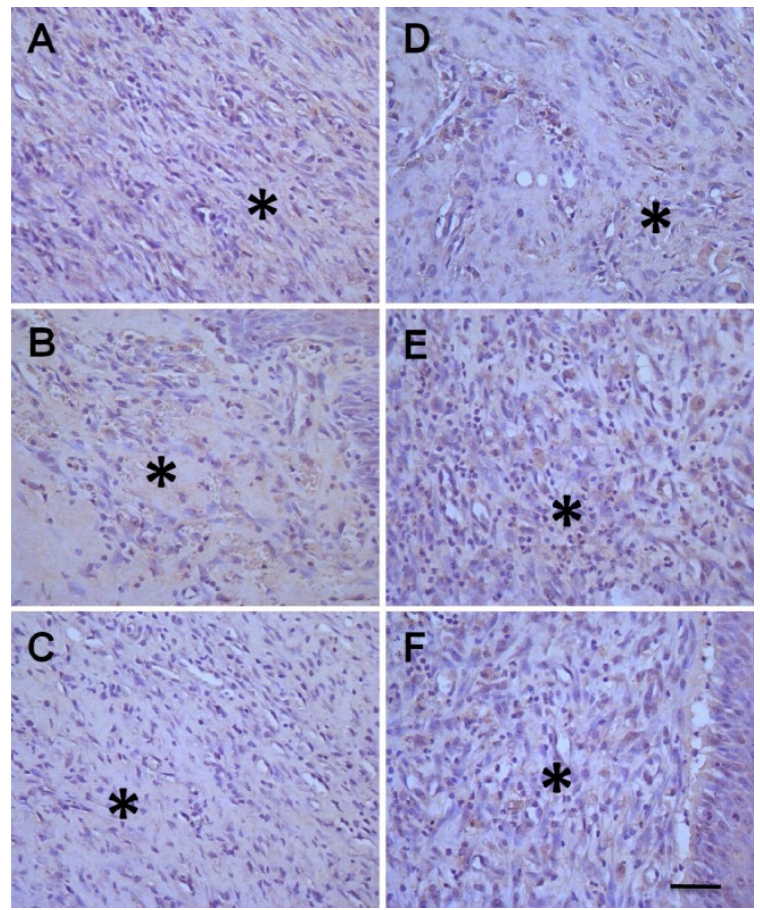

Figure 7: Histological examination of the collagen type-III expression in diabetic foot between treated and model control groups. In treated group, the model rats were treated with human mesenchymal stem cells in umbilical cord blood (hMSCs-UC) for 3 (D3), 7 (D7), and I4 days (DI4), respectively and the collagen type-III expression was examined histologically in all groups. The collagen protein type-III expression was presented as light-brown (indicated by *) under the HE staining. The left column was model control groups with D3 (A), D7 (B), and DI4 (C) and the right column was model group with hMSCs-UC for D3 (D), D7 (E), and DI4 (F). The scale bar in (F) presents 40 $\mu \mathrm{m}$ and also applied for all other images.
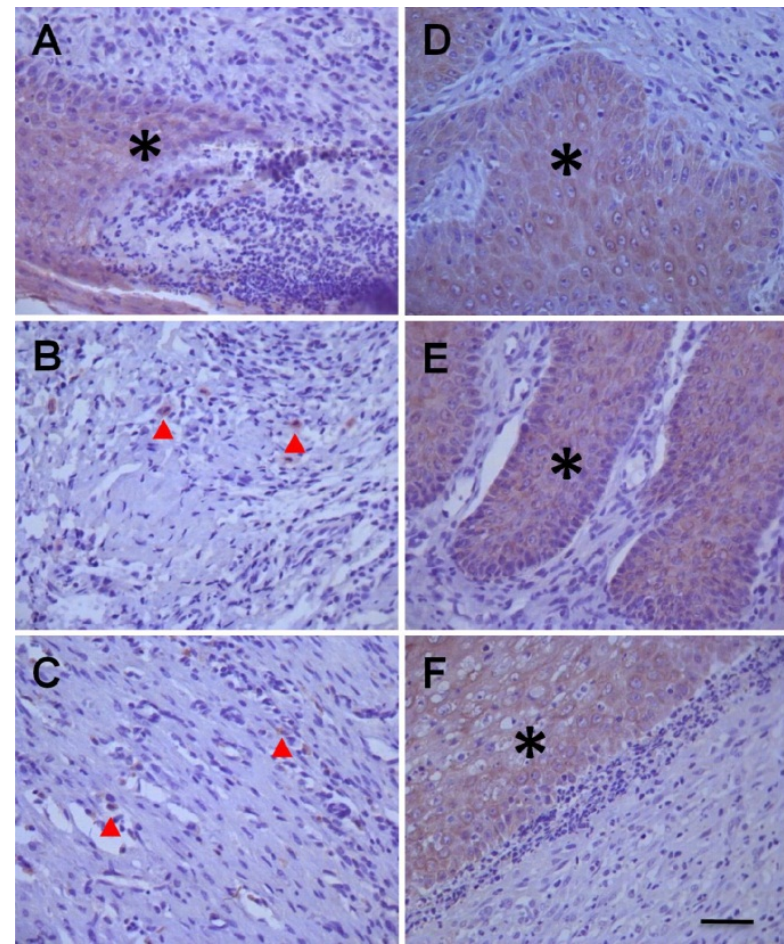

Figure 8: Histological examination of cytokeratin 19 (CKI9) expressions in diabetic foot between human mesenchymal stem cells treated diabetic rats and model control rats. The images from left column (A, B, \& C) presents the changes in CKI9 expressions at 3 (D3), 7 (D7), and I4 days (DI4) of model control rats, and the images from right column (D, E, \& F) presents the changes in CK 19 expressions at 3 (D3), 7 (D7), and 14 days (DI4) after treatment with hMSCs-UC of model rats. The mass or spot expression of CKI 9 is presented as light-brown (indicated by * or $\boldsymbol{\Delta}$ ). The scale bar in $(\mathbf{F})$ presents $40 \mu \mathrm{m}$ and also applied for all other images.

Table I: Statistical analysis of functional expression of CKI0, CKI9, Collagen type-I, and Collagen type-III in diabetic model rats before (model) and after (hMSCs) treatment with Human Mesenchymal Stem Cell from Umbilical Cord Blood (hMSCs-UC).

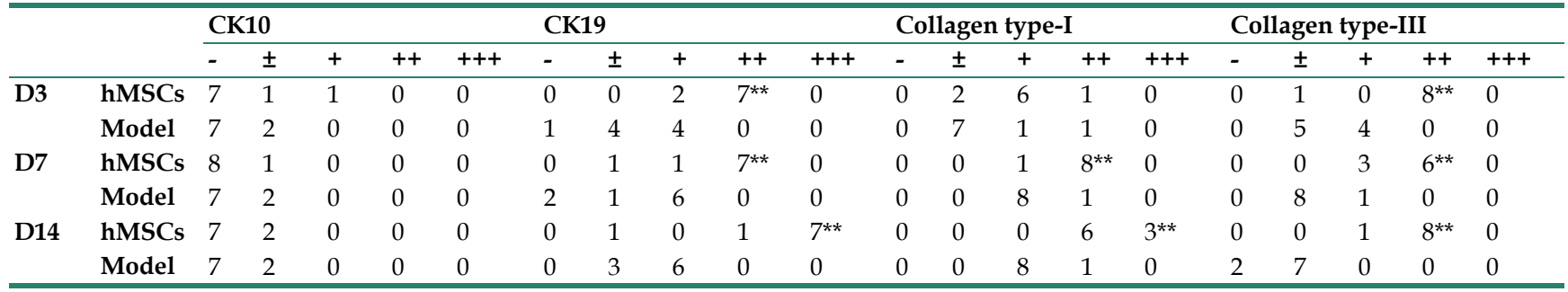

The summary data were compared between hMSCs-UC-treated model rats and model control rats with Wilcoxon rank sum test $(n=9)$. ** $P<0.01 \mathrm{vs}$ model control. Note: The expression levels of Collagen type-I, type-III, CK10, and CK19 were carefully evaluated. (-): negative expression; $(+/-)$ : positive rate of expression < 25\%; (+): positive rate of expression $<50 \%$; $(++)$ : positive rate of expression $<75 \%$; and $(+++)$ : positive rate of expression $>75 \%$. The numbers listed in this table present the total number of animals with negative or positive result in each group before and after hMSCs-UC treatment.

\section{Discussion}

\section{Major findings}

In this study, we used a well-accepted diabetic rat model in combination with treatment with human mesenchymal stem cells from umbilical cord matrix (hMSCs-UC). Using this model, we demonstrated for the first time that, within a short period of time after treatment with hMSCs-UC, these cells specifically targeted to the foot ulcerative tissue, and not to other tissues. This localization was detected by the positive expression of the human leukocyte antigen class I (HLA-I) only in the foot ulcerative tissue in these diabetic rats (Figure 4). Associated with the localization of the hMSCs-UC was significant and progressive restoration of normal tissue structure in the diabetic foot ulcers, demonstrated by a dramatic reduction in the measured size of ulceration in hMSCs-UC-treated rats compared with model control rats. The novel finding provides further evidence of targeting of hMSCs-UC treatment and is strongly supported by the finding that Wharton's jelly from umbilical cord 
blood could be transformed into a variety of cell types under certain conditions $(9,10)$. The data collected from this study are consistent with the therapeutic effects of human stem cells on myocardial infarction (11). These results also provide a practical, reliable unique method for tracking hMSCs in vivo, by detecting HLA-I as an indicator of the presence of hMSCs in animal tissues.

\section{Significance and potential clinical impact}

It has been well documented that cells from Wharton's jelly from umbilical cord blood are able to be transformed into different cell types, including adipocytes, muscular cells, epidermal cells, neurons, islet secreting cells, and even endothelial cells $(9,10)$. Because hMSCs-UC are rich, easy to collect, and proliferate up to $4 \mathrm{X}$ the number of cells within 3-5 days in appropriate culture conditions in vitro (9), it is possible that they could be produced on a large-scale. The most important advantage of hMSCs-UC is their very low Immunogenicity, which provides potential clinical benefit to patients.

Increasing evidence has shown that hMSCs-UC can differentiate into neurocyte-line cells (15-17), which is supported in this study by the expression of CD44, CD73, and CD90 in our preparations of hMSCs-UC. There is compelling evidence that hMSCs-UC may represent a promising tool in regenerative medicine, as recently demonstrated for endoderm-derived organs (such as liver) in human subjects, and also due to their immunomodulatory features compared to other MSC preparations (18). Recent published reports have confirmed the therapeutic effectiveness of hMSCs-UC in patients with myocardial infarction (11), lung disorder (19), patients with neurorestoration (20), kidney injury (21), and bone defects (22). hMSCs-UC have been utilized in a small-scale study of type 2 diabetes mellitus patients with diabetic foot disease (23) focusing on the immune disorder that accompanies diabetes. However, the majority of studies regarding hMSCs-UC are limited to clinical investigations and the underlying mechanisms by which hMSCs-UC mediated restoration of tissues have not been elucidated. In the current study, we not only explored the specific localization of hMSCs-UC in diabetic foot ulcerative tissue in a rat model of diabetes, but also further validated the potential mechanisms involved in repair of diabetic foot ulcers. We have not determined whether hMSCs-UC secrete growth factors that stimulate wound healing in diabetic foot ulceration or if they differentiate into epidermal stem cells to restore normal tissues.

\section{Human leucocyte antigen (HLA) and tracking of hMSCs-UC in vivo}

According to this data, hMSCs-UC participates in the restoration process in diabetic foot ulcers in diabetic rat model. This observation is supported by the fact that hMSCs-UC specifically localized to the targeted area in a time-dependent manner and significantly reduced the size of foot ulcers in diabetic rats treated with hMSCs-UC compared with control rats. At this point, there is still a question in this field of study as to how to effectively monitor the localization of hMSCs-UC in a targeted area over time. To answer this question, studies been performed using superparamagnetic iron oxides (SPIO) to mark hMSCs-UC in combination with an MRI technique (14); however, the MRI signal becomes weaker with the progressive differentiation of hMSCs-UC in the target tissue in the animal model, and therefore this method is not suitable for long-term observation. A recent study showed that higher dose SPIO labeling is stable for long-term MRI detection, but the labeling may limit the multi-lineage potential of hMSCs and affect their survival under serum and oxygen withdrawal (24). The daily clinical usage of gadolinium (Gd) based contrast agents in magnetic resonance imaging is limited because of its nephrogenic toxicity (25). Fluorescent dye-labeled hMSCs have a relatively low penetration capacity, and are therefore not effective for clinical use (26). In vivo tracking of hMSCs-UC has still been a technical barrier to the use of hMSCs in research and clinical investigations.

In recent reports, human leukocyte antigens (HLAs) have been used as a genetic marker to track platelet recovery and survival (27) and human migration (28). The use of HLA as a genetic marker is commonly utilized for differentiation of blood types (29). HLA may also be used as marker to track hMSCs-UC in vivo. Surprisingly, our hypothesis was well supported by the fact that human HLA from hMSCs-UC was only detected in foot ulcerative wound areas (Figure. 4) in diabetic model rats, but not in liver or kidney (Supplemental Figure 1). This finding suggests that hMSCs-UC effectively and specifically localize to the target area under these experimental conditions. In addition to localization of hMSCs-UC to the ulcerative area, the significant healing of foot ulceration in the treated rats compared with control rats is compelling evidence showing a positive effect of hMSCs-UC on diabetic foot ulcers. The use of HLA for tracking hMSCs has not been previously reported, and is a promising technique due to its antigenicity (30) and individual variability (31). However, future studies will be necessary to analyze the effectiveness of this technique. 


\section{The potential mechanisms for hMSCs-UC in wound healing}

CK19 is a protein secreted from epidermal cells located in the basal layer of skin (32). The highest expression of CK19 is in fetal basal epidermal cells and the lowest, almost negative, expression is in adults (33). CK19 is expressed in epidermal stem cells, but as the epidermal cells differentiate, the expression of CK19 is reduced and replaced by other CKs. Therefore, CK19 serves as a marker to monitor the proliferation and differentiation of epidermal stem cells (34). There is a dynamic balance between proliferation/differentiation of epidermal stem cells and the sloughing off of keratinized epidermal cells (35). CK10 can serve as a marker for mature skin because it is highly expressed in the outermost layer of keratinized epidermal cells (36), and the expression of CK10 is very weak or negative in newly repaired epidermis (37). Our observation is consistent with the above notion in that CK19 expression is increased in our model with no change in expression of CK10 (Figure 8 , Table 1), suggesting that epidermal stem cells play a role in foot ulcer repair (Figure 4). However, it remains unclear whether the healing of diabetic foot ulcers in our model is due to direct stimulation by hMSCs-UC, release of growth factors by hMSCs-UC, hMSCs-UC-mediated proliferation/differentiation of epidermal stem cells or the combination effects of both hMSCs-UC and epidermal stem cells.

Collagens distributed in the dermis (corium layer) are key players in the repair of injured skin (38). Animal studies have shown that collagen type-I and type-III are significantly increased during skin transplantation when hyaluronic acid is added to the skin graft and that the ratio of collagen I/III is dramatically decreased at the same time, which creates favorable conditions for the reconstruction of the dermis during wound healing in the skin (39). Therefore, collagen type-I and type-III may also be considered to be biomarkers for mutual and un-mutual dermis (40), respectively. Accordingly, in our study, collagen type-I and type-III were both up-regulated significantly (Figure $6 \& 5$, Table 1) in diabetic rats treated with hMSCs-UC compared with control rats and the ratio of collagen I/III was obviously reduced as described in detail in the results section. These data suggest that treatment with hMSCs-UC promotes the repair of diabetic foot ulcers.

\section{Supplementary Material}

Supplementary Figure 1

[http://www.ijbs.com/v10p0080s1.pdf]

\section{Acknowledgements}

The authors would like to thank Y. Ye and Y.
Hou from Jing-Meng Stem Cell Technology Co. Ltd. for their valuable advice on cell culture. We also thank L. Xue, K. Zhou, and C. Yang from the laboratory of the fourth affiliated hospital, Harbin Medical University for their kind help with RT-PCR, as well as S. L. Guo from the animal facility of Harbin Medical University for long term support. These authors would like also to thank BioMed Proofreading for their help in English Writing during the revision process.

\section{Funding}

This project was funded by Key Project (ZD201108) of NSF from Heilongjiang Province.

\section{Contribution statement}

QSZ, NX, NZ, ML, CLB, QZ, GFQ, and ZFC contributed to the conception and design of the study, data acquisition, analysis, and interpretation. They were all involved in writing the draft or revisions of the manuscript, and were critically important for intellectual content. All authors gave final approval to the version of the manuscript to be published. GFQ and ZFC directed the research and are the guarantors of this study.

\section{Competing Interests}

The authors have declared that no competing interest exists.

\section{References}

1. Yang W, Lu J, Weng J, Jia W, Ji L, Xiao J, et al. Prevalence of diabetes among men and women in China. N Engl J Med. 2010; 362: 1090-101.

2. Cavanagh P, Attinger C, Abbas Z, Bal A, Rojas N, Xu ZR. Cost of treating diabetic foot ulcers in five different countries. Diabetes Metab Res Rev. 2012; 28 Suppl 1: 107-11.

3. Fritschi C. Preventive care of the diabetic foot. Nurs Clin North Am. 2001; 36: 303-20.

4. Besse JL, Leemrijse T, Deleu PA. Diabetic foot: the orthopedic surgery angle. Orthop Traumatol Surg Res. 2011; 97: 314-29.

5. Ishimine H, Yamakawa N, Sasao M, Tadokoro M, Kami D, Komazaki S, et al. $\mathrm{N}$-Cadherin is a prospective cell surface marker of human mesenchymal stem cells that have high ability for cardiomyocyte differentiation. Biochem Biophys Res Commun. 2013; 438: 753-9.

6. Morigi M, Imberti B, Zoja C, Corna D, Tomasoni S, Abbate M, et al. Mesenchymal stem cells are renotropic, helping to repair the kidney and improve function in acute renal failure. J Am Soc Nephrol. 2004; 15: 1794-804.

7. Black IB, Woodbury D. Adult rat and human bone marrow stromal stem cells differentiate into neurons. Blood cells Mol Dis. 2001; 27: 632-6.

8. Jiang J, Lv Z, Gu Y, Li J, Xu L, Xu W, et al. Adult rat mesenchymal stem cells differentiate into neuronal-like phenotype and express a variety of neuro-regulatory molecules in vitro. Neurosci Res. 2010; 66: 46-52.

9. Lau TW, Lam FF, Lau KM, Chan YW, Lee KM, Sahota DS, et al. Pharmacological investigation on the wound healing effects of Radix Rehmanniae in an animal model of diabetic foot ulcer. J Ethnopharmacol. 2009; 123: 155-62.

10. Reed MJ, Meszaros K, Entes LJ, Claypool MD, Pinkett JG, Gadbois TM, et al. A new rat model of type 2 diabetes: the fat-fed, streptozotocin-treated rat. Metabolism. 2000; 49: 1390-4.

11. Zhao JY, Chai JK, Song HF, Sun TJ, Li DJ, Liu LY, et al. Effects on collagen I and III after transplantation of porcine acellular dermal matrix with hyaluronic acid. Zhonghua yi xue za zhi. 2011; 91: 1276-80.

12. Koike T, Yasuo M, Shimane T, Kobayashi H, Nikaido T, Kurita H. Cultured epithelial grafting using human amniotic membrane: the potential for using human amniotic epithelial cells as a cultured oral epithelium sheet. Arch Oral Biol. 2011; 56: 1170-6.

13. Zhang C, Fu X, Chen P, Bao X, Li F, Sun X, et al. Dedifferentiation derived cells exhibit phenotypic and functional characteristics of epidermal stem cells. J Cell Mol Med. 2010; 14: 1135-45. 
14. Yang JX, Tang WL, Wang XX. Superparamagnetic iron oxide nanoparticles may affect endothelial progenitor cell migration ability and adhesion capacity. Cytotherapy. 2010; 12: 251-9.

15. Ju XL, Huang ZW, Shi Q, Hou HS, Duan $\mathrm{CH}$. Biological characteristics and induced differentiation ability of in vitro expanded umbilical cord blood mesenchymal stem cells. Zhonghua er ke za zhi. 2005; 43: 499-502.

16. Ma L, Cui BL, Feng XY, Law FD, Jiang XW, Yang LY, et al. Biological characteristics of human umbilical cord-derived mesenchymal stem cells and their differentiation into neurocyte-like cells. Zhonghua er ke za zhi. 2006; 44: 513-7.

17. Wang LJ, Zhang YP, Wang JF, Wu YF, Xiang Y, Xie CG, et al. Biological characteristics of mesenchymal stem cells in human umbilical cord blood and their supporting capacities in ex vivo expansion of CD34+ hematopoietic stem cells. Zhonghua xue ye xue za zhi. 2005; 26: 65-8.

18. Anzalone R, Lo Iacono M, Loria T, Di Stefano A, Giannuzzi P, Farina F, et al. Wharton's jelly mesenchymal stem cells as candidates for beta cells regeneration: extending the differentiative and immunomodulatory benefits of adult mesenchymal stem cells for the treatment of type 1 diabetes. Stem Cell Rev. 2011; 7: 342-63.

19. Nystedt J, Anderson H, Tikkanen J, Pietila M, Hirvonen T, Takalo R, et al. Cell surface structures influence lung clearance rate of systemically infused mesenchymal stromal cells. Stem Cells. 2013; 31: 317-26.

20. Seo JH, Cho SR. Neurorestoration induced by mesenchymal stem cells: potential therapeutic mechanisms for clinical trials. Yonsei Med J. 2012; 53: 1059-67.

21. Fang TC, Pang CY, Chiu SC, Ding DC, Tsai RK. Renoprotective effect of human umbilical cord-derived mesenchymal stem cells in immunodeficient mice suffering from acute kidney injury. PloS One. 2012; 7: e46504.

22. Kang BJ, Ryu HH, Park SS, Koyama Y, Kikuchi M, Woo HM, et al. Comparing the osteogenic potential of canine mesenchymal stem cells derived from adipose tissues, bone marrow, umbilical cord blood, and Wharton's jelly for treating bone defects. J Vet Sci. 2012; 13: 299-310.

23. Li XY, Zheng ZH, Guo J, Zhang Y, Li H, Wang YW, et al. Treatment of foot disease in patients with type 2 diabetes mellitus using human umbilical cord blood mesenchymal stem cells: response and correction of immunological anomalies. Curr Pharm Des. 2013; 19: 4893-9.

24. Rosenberg JT, Sellgren KL, Sachi-Kocher A, Calixto Bejarano F, Baird MA, Davidson MW, et al. Magnetic resonance contrast and biological effects of intracellular superparamagnetic iron oxides on human mesenchymal stem cells with long-term culture and hypoxic exposure. Cytotherapy. 2013; 15: 307-22.

25. Neuwelt EA, Hamilton BE, Varallyay CG, Rooney WR, Edelman RD, Jacobs $\mathrm{PM}$, et al. Ultrasmall superparamagnetic iron oxides (USPIOs): a future alternative magnetic resonance (MR) contrast agent for patients at risk for nephrogenic systemic fibrosis (NSF)? Kidney Int. 2009; 75: 465-74.

26. Gouveris H, Nousia C, Giatromanolaki A, Riga M, Katotomichelakis M, Papathanasiou J, et al. Immunohistological study of wound healing after submucosal radiofrequency tissue ablation of inferior nasal turbinate in a sheep model. Am J rhinol allergy. 2011; 25: 131-6.

27. van der Meer PF, Tomson B, Brand A. In vivo tracking of transfused platelets for recovery and survival studies: an appraisal of labeling methods. Transfus Apher Sci. 2010; 42: 53-61.

28. Fernandez Vina MA, Hollenbach JA, Lyke KE, Sztein MB, Maiers M, Klitz W, et al. Tracking human migrations by the analysis of the distribution of HLA alleles, lineages and haplotypes in closed and open populations. Philos Trans R Soc Lond B Biol Sci. 2012; 367: 820-9.

29. Patel AJ, Fuentebella J, Gernez Y, Nguyen T, Bass D, Berquist W, et al. Increased HLA-DR expression on tissue eosinophils in eosinophilic esophagitis. J Pediatr Gastroenterol Nutr. 2010; 51: 290-4.

30. Ezzat T, Dhar DK, Malago M, Olde Damink SW. Dynamic tracking of stem cells in an acute liver failure model. World J Gastroenterol. 2012; 18: 507-16.

31. Caro-Oleas JL, Gonzalez-Escribano MF, Toro-Llamas S, Acevedo MJ, Martinez-Bravo MJ, Aguilera I, et al. Donor-specific antibody detection: comparison of single antigen assay and Luminex crossmatches. Tiss Antigens. 2010; 76: 398-403.

32. Lopes LB, Fabron-Jr A, Chiba AK, Ruiz MO, Bordin JO. Impact of using different laboratory assays to detect human leukocyte antigen antibodies in female blood donors. Transfusion. 2010; 50: 902-8.

33. Gennero L, De Siena R, Denysenko T, Roos MA, Calisti GF, Martano M, et al. A novel composition for in vitro and in vivo regeneration of skin and connective tissues. Cell Biochem Funct. 2011; 29: 311-33.

34. Jiang DY, Zong XL, Fu XB, Wang W, Shan F. The changing pattern of stem cell markers of sweat gland in deep partial-thickness burn wound. Zhonghua shao shang za zhi. 2009; 25: 301-4.

35. Abbas O, Richards JE, Yaar R, Mahalingam M. Stem cell markers (cytokeratin 15, cytokeratin 19 and p63) in in situ and invasive cutaneous epithelial lesions. Mod Pathol. 2011; 24: 90-7.

36. Watt FM. Epidermal stem cells as targets for gene transfer. Hum Gene Ther. 2000; 11: 2261-6.

37. Matus CE, Ehrenfeld P, Pavicic F, Sarmiento JM, Astroza A, Sanchez T, et al. Activation of kinin $\mathrm{B}$ receptor triggers differentiation of cultured human keratinocytes. Br J Dermato. 2008; 159: 792-803

38. Geburek F, Ohnesorge B, Deegen E, Doeleke R, Hewicker-Trautwein M. Alterations of epidermal proliferation and cytokeratin expression in skin biopsies from heavy draught horses with chronic pastern dermatitis. Vet Dermato. 2005; 16: 373-84

39. Cardoso CR, Favoreto S, Jr., Oliveira LL, Vancim JO, Barban GB, Ferraz DB, et al. Oleic acid modulation of the immune response in wound healing: a new approach for skin repair. Immunobiology. 2011; 216: 409-15.

40. Brown SR, Melman L, Jenkins E, Deeken C, Frisella MM, Brunt LM, et al. Collagen type I:III ratio of the gastroesophageal junction in patients with paraesophageal hernias. Surg Endosc. 2011; 25: 1390-4. 